\title{
Graphene wormholes: A condensed matter illustration of Dirac fermions in curved space
}

\author{
J. González*, J. Herrero \\ Instituto de Estructura de la Materia, Consejo Superior de Investigaciones Científicas, \\ Serrano 123, 28006 Madrid, Spain
}

Received 14 September 2009; accepted 28 September 2009

Available online 1 October 2009

\begin{abstract}
We study the properties of graphene wormholes in which a short nanotube acts as a bridge between two graphene sheets, where the honeycomb carbon lattice is curved from the presence of 12 heptagonal defects. By taking the nanotube bridge with very small length compared to the radius, we develop an effective theory of Dirac fermions to account for the low-energy electronic properties of the wormholes in the continuum limit, where the frustration induced by the heptagonal defects is mimicked by a line of fictitious gauge flux attached to each of them. We find in particular that, when the effective gauge flux from the topological defects becomes maximal, the zero-energy modes of the Dirac equation can be arranged into two triplets, that can be thought as the counterpart of the two triplets of zero modes that arise in the dual instance of the continuum limit of large spherical fullerenes. We further investigate the graphene wormhole spectra by performing a numerical diagonalization of tight-binding Hamiltonians for very large lattices realizing the wormhole geometry. The correspondence between the number of localized electronic states observed in the numerical approach and the effective gauge flux predicted in the continuum limit shows that graphene wormholes can be consistently described by an effective theory of two Dirac fermion fields in the curved geometry of the wormhole, opening the possibility of using real samples of the carbon material as a playground to experiment with the interaction between the background curvature and the Dirac fields.
\end{abstract} (c) 2009 Elsevier B.V. All rights reserved.

PACS: 73.20.-r; 73.22.-f; 04.62.+v

Keywords: Graphene

\footnotetext{
* Corresponding author.

E-mail address: gonzalez@iem.cfmac.csic.es (J. González).
} 


\section{Introduction}

In recent years, the feasibility of fabricating in the laboratory individual sheets of graphite (so-called graphene) has opened an exciting field of research in condensed matter physics [1-3]. This new material illustrates the case of a genuine two-dimensional electron system, with remarkable electronic properties and great potential for technological applications [4]. Many of the interesting features of graphene arise actually from the unconventional band structure of the carbon sheet. The undoped material has conical conduction and valence bands that meet at the corners of the hexagonal Brillouin zone, thus mimicking at low energies the dispersion of electrons and positrons in the Dirac theory (though with a Fermi velocity about 300 times smaller than the speed of light). There have been already clear experimental signatures of the Dirac-like dispersion of the electron system as, for instance, the peculiar sequence of plateaus measured in the quantum Hall effect [2,3]. The relativistic invariance of the low-energy theory is also at the origin of many of the outstanding properties of graphene, like the insensitivity of the electronic transport to scatterers with size larger than the lattice spacing [5] or the transparent transmission at normal incidence through potential barriers [6].

Part of the great attention received by the two-dimensional carbon material has come from the possibility of observing phenomena in such an electron system which otherwise would be confined to the realm of high-energy physics. Under usual experimental conditions, the interacting theory is in the strong-coupling regime, what has led to propose effects like the hyper-critical screening of charged impurities [7-10], or quasiparticle features deriving from the renormalization of the interaction in the many-body theory [11-13].

In this promising picture of graphene, there are however other aspects that still have to be better understood. One of them is the role played by the curvature of the two-dimensional system. The experimental samples of graphene always show some corrugation, that is due in part to the irregularities in the surface of the substrate on which they are deposited. However, suspended samples also have ripples, which is a clear indication of the natural tendency of graphene to develop a modulation of the two-dimensional geometry. This effect translates into a small spatial dependence of the electron hopping between carbon atoms, that can be simulated by the introduction of fictitious gauge fields [14]. These are characterized anyhow by having a vanishing flux through the two-dimensional system on the average, as long as the graphene sheet does not bend over macroscopic length scales.

In this paper we will be interested in the effects of the curvature on graphene in cases where that is strong enough to modify the topology of the carbon lattice. The deviation from a flat twodimensional geometry over large length scales implies that the intrinsic curvature cannot vanish everywhere, and this is only possible through the appearance of carbon rings that are not sixmembered within the bulk hexagonal lattice of the graphene sheet. The most notorious instance where these so-called topological defects play an important role is provided by the fullerenes, where 12 pentagonal carbon rings are just enough to close the carbon sheet into a spherical cage, no matter the size of the molecule [15]. The carbon lattices we are going to investigate here are to some extent dual to the fullerenes, as the carbon sheet is bent now through the introduction of heptagonal carbon rings. The two-dimensional geometries have then some content of negative curvature, describing the matching between different infinite spaces. In this respect, the geometry of a carbon nanotube-graphene junction has been already studied in Ref. [16]. Here we will focus on the case where the carbon sheet is curved more strongly, to give rise to a wormhole bridge between two different graphene branches. 
The duality between the fullerene and wormhole geometries becomes more explicit after noticing that 12 heptagonal carbon rings are needed to establish the connection between the two graphene branches of the wormhole. The presence of the topological defects has in general important consequences regarding the electronic properties of the carbon material. It has been shown that the frustration that they induce in the hexagonal carbon lattice can be mimicked by the action of fictitious gauge fields on the electron system [15]. Opposite to the case of the gauge fields introduced for the ripples, the fields related to the topological defects imply a nonvanishing flux threading each odd-membered carbon ring. This means that the structure of the low-lying electronic levels is in general dictated by the number of topological defects in the carbon lattice, as illustrated in the case of the fullerenes $[15,17]$. The number of localized states in the carbon nanotube-graphene junctions has also shown to be related to the distribution of heptagonal carbon rings at the junction [16]. Here we will show that, in the graphene wormholes with maximal gauge flux, the localized states can be arranged into two triplets that are the counterpart of the zero-energy modes of the fullerene lattices in the continuum limit.

The content of the paper is distributed as follows. In Section 2, we work out a simplified continuum model of the wormhole geometry. We review next in Section 3 the correspondence between fictitious gauge fields and odd-membered rings in the carbon lattice. Section 4 is devoted to set up the effective low-energy theory of Dirac fermions interacting with the fictitious gauge field in the curved geometry of the wormhole, showing the correlation between the effective gauge flux and the number of localized states in the electron system. The numerical investigation of graphene wormhole spectra is described in Section 5, where a comparison with the results of the continuum field theory is also carried out. Finally, the conclusions of our work are drawn in Section 6 .

\section{Wormhole geometry}

A graphene layer can be curved into a carbon nanotube from the presence of heptagonal carbon rings at the base of the junction. It can be shown that the number of heptagonal rings needed is always 6, provided that there are no other topological defects [16]. A graphene wormhole consists of a short nanotube bridging two different graphene layers. This can be viewed then as two nanotube-graphene junctions connected at the end of each nanotube. We will show that the number of topological defects implied by this construction is consistent with the value of the Euler characteristic computed for the geometry that arises in the continuum limit of the graphene wormhole.

The simplest way of formally building a nanotube-graphene junction follows the three-step process represented in Fig. 1. One can make first a hole with hexagonal shape in a graphene layer (Fig. 1(a)). New vertical bonds can be attached then to the atoms at the boundary of the hole, to produce next a zig-zag pattern connecting new atoms at the other end of the vertical bonds (Fig. 1(b)). Thus, the lattice is already prepared to be extended with a zig-zag nanotube away from the hole (Fig. 1(c)). We see that 6 heptagonal rings are introduced at step 2 of this construction, alternating with hexagonal rings at the boundary of the hole. It is clear that the procedure can be generalized to attach zig-zag nanotubes of larger radius, while the number of heptagonal rings required is always the same. In what follows we will have in mind those graphene wormholes where the topological defects are regularly distributed along the base of each nanotube-graphene junction. The zig-zag nanotubes that can be attached in this way may have a number of $6 n$ hexagons along the waist, corresponding to a $(6 n, 0)$ chirality in the conventional notation to denote the carbon nanotubes [18]. 


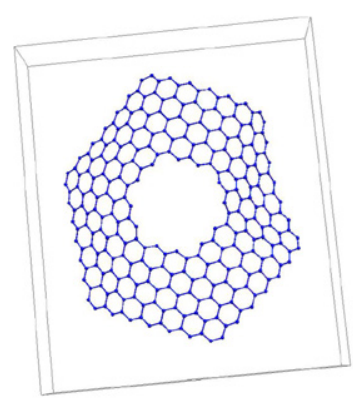

(a)

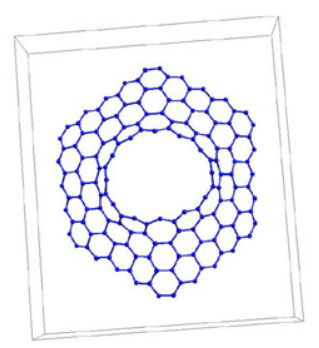

(b)

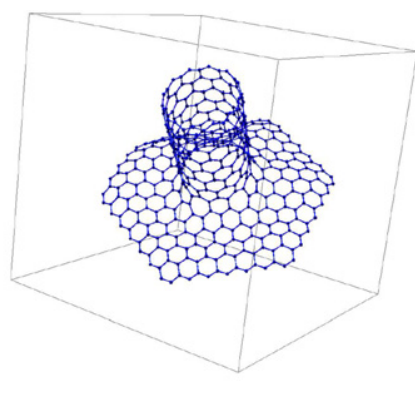

(c)

Fig. 1. Three steps in the construction of a carbon nanotube-graphene junction, where it is shown the appearance of the 6 heptagonal carbon rings at the base of the junction.

We will take the continuum limit of the graphene wormhole by making the radius $R$ of the nanotube much larger than its length. In this way, the wormhole will appear at large distance scales as the surface of two planes connected at the boundary of a common hole. This is the minimal model required to describe the geometry of the wormhole, as it disregards possible smooth variations of the curvature in the region between the planes. We will see however that such a description already contains all the factors needed to reproduce the topological features of the Dirac theory in the curved space.

In the continuum model of the wormhole geometry, we may start with two coordinate charts to cover the two branches (lower and upper plane) of the two-dimensional manifold. For each of the two charts we may use polar coordinates, that we will denote respectively as $r_{-}, \theta_{-}$and $r_{+}, \theta_{+}$, with the constraint $r_{-}, r_{+} \geqslant R$. There is however an obvious way of mapping the upper branch with $r_{+} \geqslant R$ into the region corresponding to the hole in the lower branch. We can make the definition

$$
r_{-}=\frac{R^{2}}{r_{+}}, \quad r_{+} \geqslant R
$$

to extend the values of $r_{-}$below $R$. In this way, we can pass to describe the two-dimensional manifold by means of a single chart, with $0<r_{-}<\infty$. The line element $d s$ is given in the region outside the circle $r_{-}=R$ by the conventional metric

$$
d s^{2}=d r_{-}^{2}+r_{-}^{2} d \theta_{-}^{2}, \quad r_{-} \geqslant R
$$

while in the inner region it becomes

$$
d s^{2}=\left(\frac{R}{r_{-}}\right)^{4}\left(d r_{-}^{2}+r_{-}^{2} d \theta_{-}^{2}\right), \quad r_{-} \leqslant R
$$

It is reassuring to see that the metric thus defined is continuous at $r_{-}=R$ (what would not have been the case if a higher negative power had been used in the change of variables (1)). The first derivative of the metric tensor is however discontinuous, and this is the reflection of the curvature that the wormhole concentrates at the junction between the two planes.

From now on we will drop the lower label in the coordinates $r_{-}, \theta_{-}$, so that we will be using a single radial coordinate with $0<r<\infty$. In this single chart, the metric tensor is given by

$$
g_{\mu \nu}=\Omega^{2}(r)\left(\begin{array}{cc}
1 & 0 \\
0 & r^{2}
\end{array}\right)
$$


with the conformal factor

$$
\Omega(r)=\left(\frac{R}{r}\right)^{2} \theta(R-r)+\theta(r-R)
$$

From the metric tensor, we obtain the nonvanishing components of the connection

$$
\begin{aligned}
& \Gamma_{r r}^{r}=\frac{\Omega^{\prime}(r)}{\Omega(r)} \\
& \Gamma_{\theta \theta}^{r}=-r^{2} \frac{\Omega^{\prime}(r)}{\Omega(r)}-r \\
& \Gamma_{r \theta}^{\theta}=\frac{\Omega^{\prime}(r)}{\Omega(r)}+\frac{1}{r}
\end{aligned}
$$

One finds that the dependence on $r$ of the above objects is simply proportional to step functions. Hence, the curvature tensor turns out to be proportional to a delta function centered at $r=R$. We get the nonvanishing component

$$
\begin{aligned}
\mathcal{R}_{\theta r \theta}^{r} & =-r^{2} \frac{\Omega^{\prime \prime}(r)}{\Omega(r)}+r^{2}\left(\frac{\Omega^{\prime}(r)}{\Omega(r)}\right)^{2}-r \frac{\Omega^{\prime}(r)}{\Omega(r)} \\
& =-2 R \delta(r-R)
\end{aligned}
$$

From this result, we get finally the scalar curvature

$$
\mathcal{R}=-\frac{4}{R} \delta(r-R)
$$

In our minimal model for the wormhole geometry, the curvature is thus localized at the contact region between the lower and the upper plane. This is the consequence of having taken a continuum limit that compresses the bridge of the wormhole to a circle. This description is however useful, as it allows us to establish a correspondence with the amount of curvature localized at the topological defects in the carbon lattice. Although the two-dimensional space considered here is infinite, we can still compute its Euler characteristic, given the compact support of the scalar curvature. Thus, we get

$$
\begin{aligned}
\chi & =\frac{1}{4 \pi} \int d^{2} x \sqrt{\operatorname{det}(g)} \mathcal{R} \\
& =-\frac{1}{4 \pi} \int d r d \theta 4 \delta(r-R)=-2
\end{aligned}
$$

We note that the same result is obtained when applying the expression of the Euler characteristic for the simplex defined by the carbon lattice of the wormhole. The curvature is localized there in the heptagonal carbon rings, in the same way as it is so for the pentagonal rings of fullerene molecules [15]. In the case of the fullerenes, no matter the size, 12 five-fold rings are just needed to curve the carbon lattice into a spherical cage. As long as the content of curvature of a pentagonal ring is the opposite to that of a heptagonal ring, we can assert that a geometry with 12 heptagonal rings must have an Euler characteristic equal to -2, which is the opposite to that of the sphere. This is consistent with the result (13) obtained for the two-dimensional surface, showing that our simplified geometry makes sense as a description of the graphene wormhole in the continuum limit. 


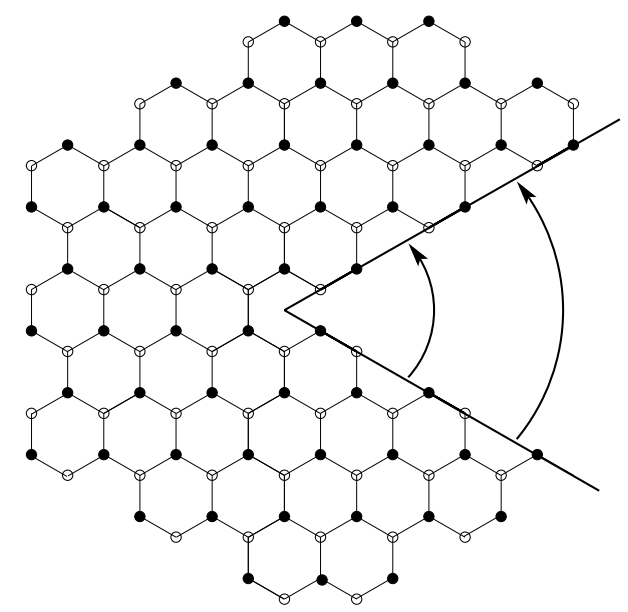

Fig. 2. Formal construction of a pentagonal carbon ring in a honeycomb graphene lattice.

\section{Gauge fields from topological defects}

In order to set up the correct continuum theory of graphene wormholes, we have to take into consideration that the effect of the topological defects is not only to curve the graphene lattice. This was first realized when looking at the low-energy electronic levels of large fullerene molecules. Tight-binding calculations carried out in a series of growing clusters showed that a sensible continuum limit should account for the existence of two triplets of zero-energy modes in the electronic spectrum [17]. It turned out that such a degeneracy can be actually understood as the effect of a fictitious gauge field on the curved molecule, arising from the particular transformation induced by the disclinations on the electron fields [15].

We start reviewing the nontrivial role of the topological defects by recalling that the honeycomb graphene lattice has two atoms in the unit cell, in such a way that the material is made of two interpenetrating sublattices corresponding to the black and white atoms shown in Fig. 2. Each carbon atom contributes with one electron to the transport properties, and the tight-binding Hamiltonian can therefore be written in real space as

$$
H=-t \sum_{\langle i, j\rangle} c_{\bullet i}^{\dagger} c_{\circ j}-t \sum_{\langle i, j\rangle} c_{\circ i}^{\dagger} c_{\bullet j}
$$

where $c_{\bullet i}^{\dagger}, c_{\circ i}^{\dagger}\left(c_{\bullet i}, c_{\circ i}\right)$ are electron creation (annihilation) operators in the two different sublattices, and the sum runs over pairs of nearest-neighbor carbon atoms.

When passing to momentum space, the diagonalization of the tight-binding Hamiltonian leads to the eigenvalue problem

$$
\left(\begin{array}{cc}
0 & -t \sum_{j} e^{i \mathbf{p} \cdot \mathbf{u}_{j}} \\
-t \sum_{j} e^{-i \mathbf{p} \cdot \mathbf{u}_{j}} & 0
\end{array}\right)\left(\begin{array}{l}
\psi_{\bullet} \\
\psi_{\circ}
\end{array}\right)=\varepsilon(\mathbf{p})\left(\begin{array}{l}
\psi_{\bullet} \\
\psi_{\circ}
\end{array}\right)
$$

where $\left\{\mathbf{u}_{j}\right\}$ is the set of vectors connecting a carbon atom to its nearest neighbors. We obtain from (15) the conduction and valence bands of graphene

$$
\varepsilon(\mathbf{p})= \pm t \sqrt{1+4 \cos ^{2}\left(\frac{\sqrt{3}}{2} p_{x} a\right)+4 \cos \left(\frac{\sqrt{3}}{2} p_{x} a\right) \cos \left(\frac{3}{2} p_{y} a\right)}
$$




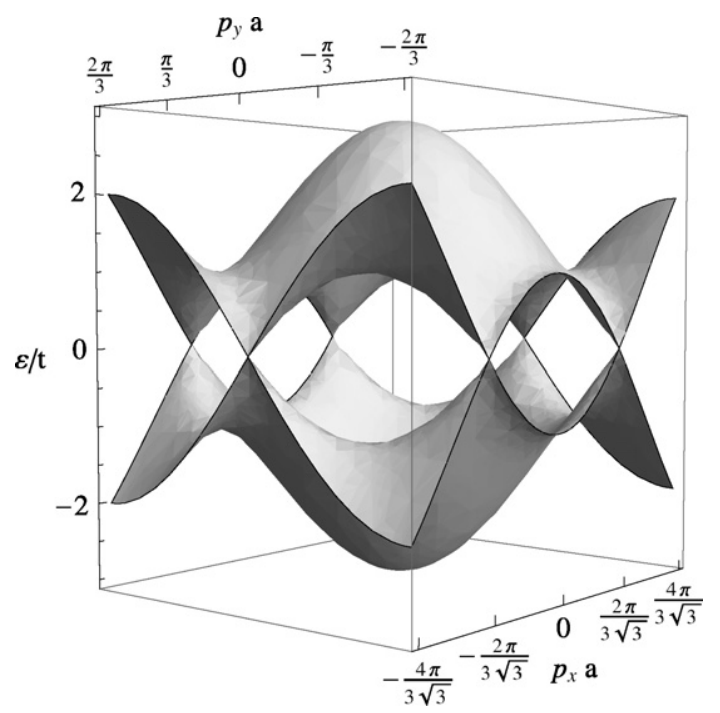

Fig. 3. Plot of the dispersion (16) displaying the conical shape of the valence and conduction bands at the corners of the hexagonal Brillouin zone.

where $a$ stands for the $\mathrm{C}-\mathrm{C}$ distance.

The Fermi level of the undoped system is at $\varepsilon=0$. The expansion of (16) about this level shows that the conduction and valence bands have conical shape at low energies, meeting at isolated Fermi points (so-called Dirac points) which coincide with the corners of the hexagonal Brillouin zone (see Fig. 3). Given the equivalence relation that exists between different points in momentum space, it happens that all the low-energy states can be accommodated into the conical bands around two inequivalent Dirac points, at corners $K$ and $K^{\prime}$ of the Brillouin zone that can be chosen to make an angle of $\pm \pi / 3$ or $\pi$ [15]. This implies that the continuum limit of the electron system is given in general by the theory of two Dirac fermion fields encoding all the low-energy electronic excitations.

We observe now that the presence of pentagonal or heptagonal rings leads to frustration in the carbon lattice, in the sense that the atoms cannot be distributed then in two different sublattices. We can formally construct a pentagonal defect in the honeycomb lattice by cutting off a sector of 60 degrees and matching next the two boundaries of the remaining space, as represented in Fig. 2. In this operation, it is clear that one is identifying pairs of carbon atoms that belonged to different sublattices in the original graphene sheet. In the continuum limit, the rotation of 60 degrees needed to bring one of the boundaries of the cut graphene plane onto the other can be properly implemented by introducing a suitable spin connection, realizing the parallel transport of the fields when going around the topological defect [15].

There is however an additional effect from the above construction that requires separate consideration, as the rotation of 60 degrees leads to a transformation in momentum space that maps each inequivalent Dirac point into the other. That is, the action of matching the two boundaries in Fig. 2 leads to the exchange of corresponding electron states at the two different Dirac valleys. The imaginary line that signals the cut in the original graphene plane is just an artifact to define that operation, as the exchange of the electron states is only realized when making a complete turn around the topological defect. This means that the transformation can be regarded 
as that corresponding to the parallel transport by a gauge field whose flux is concentrated at the defect [15].

A careful consideration of the mapping of the two different Dirac valleys under a rotation of 60 degrees shows that the transformation acts on the wavefunctions of the corresponding electron states at the two Dirac points $K$ and $K^{\prime}$ as

$$
\left(\begin{array}{c}
\psi_{K}^{\prime} \\
\psi_{K^{\prime}}^{\prime}
\end{array}\right)=\left(\begin{array}{cc}
0 & 1 \\
-1 & 0
\end{array}\right)\left(\begin{array}{c}
\psi_{K} \\
\psi_{K^{\prime}}
\end{array}\right)
$$

The transformation (17) can be written as the action of a $S U(2)$ gauge field $\mathbf{A}$ on the electrons turning around the topological defect. In polar coordinates, the vector potential must have $A_{\theta}$ as its only nonvanishing component, fixed by the condition that for any closed path $C$ containing the topological defect

$$
e^{i \oint_{C} d \theta A_{\theta}}=\left(\begin{array}{cc}
0 & 1 \\
-1 & 0
\end{array}\right)
$$

The potential $\mathbf{A}$ is valued on two-dimensional hermitian matrices, and it can be viewed as a particular configuration of a non-Abelian gauge field. We find that

$$
A_{\theta}=\frac{\Phi}{2 \pi} \tau_{2}
$$

where $\tau_{2}$ is taken from a set of sigma matrices $\left\{\tau_{i}\right\}$, and $\Phi=\pi / 2$.

We observe then that the frustration induced by the pentagonal (as well as heptagonal) carbon rings can be mimicked by attaching a line of fictitious gauge flux $\Phi$ at the topological defect. The corresponding gauge field acts on the space of the two Dirac points and accounts in general for important effects in the low-energy part of the electronic spectrum. In particular, it plays a crucial role to establish the correct number of zero-energy modes of the electron system, as shown in the case of the series of growing fullerene molecules [15].

In what follows, we will need to represent the effect of several heptagonal rings on the wormhole geometry. A relevant question is that their combined action does not lead in general to a gauge field corresponding to the sum of the fluxes of the individual defects. This is so as the above representation (18) is referred to a particular center of rotation. The case of two close pentagonal rings has been analyzed in detail in Ref. [19], making clear that the assessment of their global effect requires bringing the action of the respective transformations to a common frame. This implies a translation in the honeycomb lattice, that does not commute with the action given by (18). The consequence is that the flux felt away from two topological defects adds to $\pi$ only when the distance between them is given by a vector $(N, M)$ (in units of the translation vectors of the honeycomb lattice) such that $N-M$ is a multiple of 3 [19]. In the rest of the cases, the total effective flux turns out to be given by $\pi / 3$. For our purposes, it will be enough to use this result to compute the global effect of the 6 heptagonal carbon rings at each side of the wormhole bridge, and to check that their relative location is in correspondence with the number of zero-energy modes in the spectrum.

\section{Continuum limit of graphene wormholes}

\subsection{Effective field theory}

Our aim is to describe the low-energy regime of the electronic spectrum of graphene wormholes, taking the limit in which the radius $R$ of the wormhole bridge is much larger than the lattice 
spacing. The energy levels have to correspond then to the eigenvalues of the system of two Dirac fermion fields on the wormhole geometry, feeling also the fictitious gauge flux attached to the topological defects. Regarding this latter effect, we remark that, while the vector potential (19) represents a $S U$ (2) connection, its action can be made effectively Abelian by passing to the basis of eigenvectors of $\tau_{2}$. We end up in this way with two different Dirac equations for respective fields $\Psi^{+}$and $\Psi^{-}$, corresponding to the two different orientations (outgoing and ingoing) of the fictitious gauge flux through the surface of the wormhole. We write then the two eigenvalue problems as

$$
i v_{F} \boldsymbol{\sigma} \cdot(\nabla \mp i \mathbf{A}) \Psi^{ \pm}=\varepsilon \Psi^{ \pm}
$$

$v_{F}$ being the Fermi velocity and $\pm \mathbf{A}$ the Abelian component of the vector potential after projection onto the eigenvectors of $\tau_{2}$ (we work in units such that $\hbar=1$ ).

As long as we are interested in the long-wavelength solutions of (20), it will be justified to approximate the effect of the fictitious gauge field by its action far away from the wormhole bridge, where the flux coming from the topological defects is felt isotropically. Thus, we will assume in the resolution of (20) that the vector potential can be taken with a constant nonvanishing component $A_{\theta}=\Phi / 2 \pi$. Anyhow, we know that the effective flux $\Phi$ has to be consistent with the combined action of the topological defects around the wormhole bridge. According to what has been stated at the end of the preceding section, such an effective flux may not correspond in general to the simple addition of individual fluxes from the defects, but it can be precisely computed from their relative position in the wormhole.

As usual for the wave equation of a fermion field, all the vectors in (20) are referred to the tangent space at each point of the two-dimensional surface. The passage to derivatives with respect to the coordinates $x_{\mu}$ is achieved by making use of the zweibein $e_{a}^{\mu}$, defined in terms of the metric tensor $g_{\mu \nu}$ by

$$
g_{\mu \nu} e_{a}^{\mu} e_{b}^{\nu}=\delta_{a b}
$$

The covariant derivative in (20) can be written as $\nabla_{\mu}=\partial_{\mu}+\Gamma_{\mu}$, where the spin connection is given by [20]

$$
\Gamma_{\mu}=\frac{1}{8}\left[\sigma^{a}, \sigma^{b}\right] e_{a}^{\nu} \nabla_{\mu} e_{b \nu}
$$

For the particular coordinate system of Section 2, it is easily found that

$$
\begin{aligned}
& \Gamma_{r}=0 \\
& \Gamma_{\theta}=-\frac{i}{2} \sigma_{3}\left(r \frac{\Omega^{\prime}(r)}{\Omega(r)}+1\right)
\end{aligned}
$$

More explicitly, $\Gamma_{\theta}$ turns out to have a discontinuity at $r=R$, from $-(i / 2) \sigma_{3}$ in the outer region of the circle to $(i / 2) \sigma_{3}$ in the inner region.

Taking into account the form of the spin connection (24) and the gauge connection (19), we can split the Dirac equation into two different expressions corresponding to the lower and the upper sheet of the wormhole:

$$
\begin{aligned}
& i v_{F}\left(\begin{array}{cc}
0 & \partial_{r}-\frac{1}{r} i \partial_{\theta} \mp \frac{\Phi}{2 \pi r}+\frac{1}{2 r} \\
\partial_{r}+\frac{1}{r} i \partial_{\theta} \pm \frac{\Phi}{2 \pi r}+\frac{1}{2 r} & 0
\end{array}\right)\left(\begin{array}{l}
\Psi_{A}^{ \pm} \\
\Psi_{B}^{ \pm}
\end{array}\right)=\varepsilon\left(\begin{array}{l}
\Psi_{A}^{ \pm} \\
\Psi_{B}^{ \pm}
\end{array}\right) \\
& \quad \text { for } r \geqslant R
\end{aligned}
$$




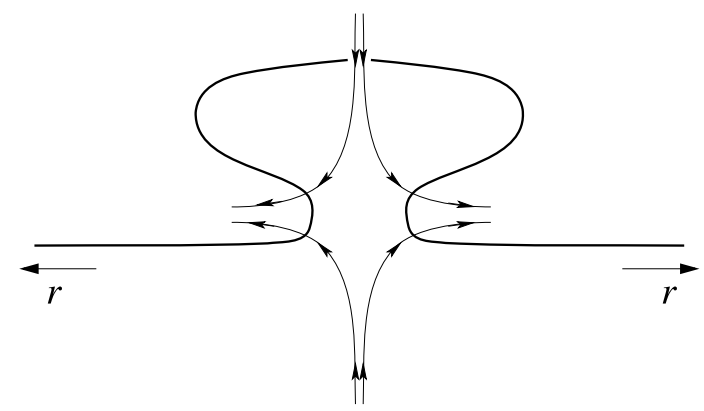

Fig. 4. Schematic representation of the section of a wormhole in the process of compactification of the upper sheet into the inner region of the circle $r \leqslant R$. The oriented lines correspond to the fictitious gauge flux threading the topological defects.

and

$$
\begin{aligned}
& i v_{F}\left(\frac{r}{R}\right)^{2}\left(\begin{array}{cc}
0 & \partial_{r}-\frac{1}{r} i \partial_{\theta} \mp \frac{\Phi^{\prime}}{2 \pi r}-\frac{1}{2 r} \\
\partial_{r}+\frac{1}{r} i \partial_{\theta} \pm \frac{\Phi^{\prime}}{2 \pi r}-\frac{1}{2 r} & 0
\end{array}\right)\left(\begin{array}{c}
\Psi_{A}^{ \pm} \\
\Psi_{B}^{ \pm}
\end{array}\right)=\varepsilon\left(\begin{array}{c}
\Psi_{A}^{ \pm} \\
\Psi_{B}^{ \pm}
\end{array}\right) \\
& \quad \text { for } r \leqslant R
\end{aligned}
$$

In the above equations, we can think of $\Psi_{A}$ and $\Psi_{B}$ as the respective amplitudes of the electron in the two sublattices of the graphene lattice, assuming that such a division of the carbon atoms makes sense away from the wormhole bridge.

In writing (25) and (26), we have introduced different effective fluxes $\Phi$ and $\Phi^{\prime}$ felt by electrons turning respectively in the outer and the inner region of the circle $r=R$. The discussion about the actual value of these fluxes is rather subtle, as in order to deal with a single coordinate $r$ we have mapped the upper graphene sheet into the closed region with $r \leqslant R$. Now the electrons turning in this inner region may only feel an effective flux threading the origin at $r=0$, that corresponds to the flux escaping to the point at infinity from the original upper sheet. This flux comes from the 6 topological defects at the junction with the wormhole bridge, as represented schematically in Fig. 4. This analysis shows that, while $\Phi$ has to be effectively the flux induced by the 6 topological defects at the junction in the lower graphene sheet, $\Phi^{\prime}$ must be equal to the opposite flux $-\Phi$ threading the origin at $r=0$.

The preceding argument can be confirmed by reverting the change of variables (1) to write Eq. (26) in the original coordinate $r_{+}>R$ of the upper graphene branch. According to the above discussion, Eq. (26) actually reads

$$
\begin{aligned}
& i v_{F}\left(\frac{r}{R}\right)^{2}\left(\begin{array}{cc}
0 & \partial_{r}-\frac{1}{r} i \partial_{\theta} \pm \frac{\Phi}{2 \pi r}-\frac{1}{2 r} \\
\partial_{r}+\frac{1}{r} i \partial_{\theta} \mp \frac{\Phi}{2 \pi r}-\frac{1}{2 r} & 0
\end{array}\right)\left(\begin{array}{l}
\Psi_{A}^{ \pm} \\
\Psi_{B}^{ \pm}
\end{array}\right)=\varepsilon\left(\begin{array}{c}
\Psi_{A}^{ \pm} \\
\Psi_{B}^{ \pm}
\end{array}\right) \\
& \quad \text { for } r \leqslant R
\end{aligned}
$$

By passing to the variable $r_{+}=R^{2} / r$, we obtain

$$
\begin{aligned}
& -i v_{F}\left(\begin{array}{cc}
0 & \partial_{r_{+}}+\frac{1}{r_{+}} i \partial_{\theta} \mp \frac{\Phi}{2 \pi r_{+}}+\frac{1}{2 r_{+}} \\
\partial_{r_{+}}-\frac{1}{r_{+}} i \partial_{\theta} \pm \frac{\Phi}{2 \pi r_{+}}+\frac{1}{2 r_{+}} & 0
\end{array}\right)\left(\begin{array}{l}
\Psi_{A}^{ \pm} \\
\Psi_{B}^{ \pm}
\end{array}\right)=\varepsilon\left(\begin{array}{c}
\Psi_{A}^{ \pm} \\
\Psi_{B}^{ \pm}
\end{array}\right) \\
& \text {for } r_{+} \geqslant R
\end{aligned}
$$

It is clear that Eq. (28) is formally identical to (25), up to a parity transformation accounting for the different orientation of the angular variable $\theta$. This simply comes from the fact that, when 
the wormhole is obtained by matching two similar graphene branches, the rotation around it is seen as clockwise in one of the copies and counter-clockwise in the other.

In the subsequent discussion, we will need to make a precise assignment for the flux $\Phi$ induced by the 6 topological defects at the junction of each graphene sheet with the wormhole bridge. As already stated, the total flux is dictated by the relative distance $(N, M)$ between heptagonal carbon rings, in such a way that all graphene wormholes can be classified in two different classes, depending on whether the value of $N-M$ is a multiple of 3 or not.

\subsection{Zero-energy modes}

We address here the possibility of having bound states in the resolution of the Dirac equation (20). In the case of massive Dirac fermions, such localized states correspond to energy levels within the gap of the spectrum. As we are dealing with massless fermions, the search for bound states has to concentrate on eigenmodes with $\varepsilon=0$. On the other hand, the number of these zero-energy modes is in general a measure of the gauge flux traversing a two-dimensional system. We will see that, in our case, the existence of the fictitious gauge field provided by the topological defects is what gives rise to a certain number of states bound to the wormhole bridge.

In order to determine the effective flux $\Phi$ that appears in Eqs. (25) and (27), we have to pay attention to the details of the lattice forming the wormhole. As already stated, we will focus here on geometries where the topological defects are regularly distributed at both ends of the wormhole bridge. When this consists of a small piece of zig-zag nanotube, the construction represented in Fig. 1 shows that we can have in general a number $6 n$ of hexagonal carbon rings around the waist of the tube. This means that the relative distance between heptagonal rings will be given then by the couple $(n, 0)$, following the usual notation in units of the translation vectors of the honeycomb lattice [18]. According to the results of Ref. [19], it is only when $n$ is a multiple of 3 that the effective flux from the 6 heptagonal rings at each graphene branch corresponds to the sum of the individual fluxes. In that instance, we will have $\Phi=3 \pi$. When $n$ is not a multiple of 3 , the combined flux of each pair of heptagons will only add to $\pi / 3$, giving a total flux $\Phi=\pi$ for the 6 topological defects at each graphene branch. We will discuss then separately these two classes of wormhole geometries in the computation of the zero modes of the Dirac equation.

We first consider the case in which the total flux is $\Phi=3 \pi$. Setting $\varepsilon=0$ in Eqs. (25) and (27), we observe that the eigenmodes get in general a power-law dependence as a function of $r$. Choosing for instance a solution with $\Psi_{A}^{ \pm}=0$, we have the equations

$$
\left(\partial_{r}-\frac{1}{r} i \partial_{\theta} \mp \frac{3}{2 r}+\frac{1}{2 r}\right) \Psi_{B}^{ \pm}=0 \quad \text { for } r \geqslant R
$$

and

$$
\left(\partial_{r}-\frac{1}{r} i \partial_{\theta} \pm \frac{3}{2 r}-\frac{1}{2 r}\right) \Psi_{B}^{ \pm}=0 \quad \text { for } r \leqslant R
$$

Looking now for solutions that decay at both $r \rightarrow \infty$ and $r \rightarrow 0$, we find suitable behaviors for $\Psi_{B}^{-}$

$$
\begin{aligned}
& \Psi_{B}^{-} \sim r^{-l-2} e^{i l \theta}, \quad r \geqslant R \\
& \Psi_{B}^{-} \sim r^{-l+2} e^{i l \theta}, \quad r \leqslant R
\end{aligned}
$$


It is clear then that these modes lead to states localized at the wormhole for $l=0$ and \pm 1 . A more detailed inspection shows however that only the state with $l=0$ is strictly normalizable. In the curved space, the finiteness of the norm of the eigenstates is enforced by the condition

$$
\int d^{2} x \sqrt{\operatorname{det}(g)}|\Psi(\mathbf{r})|^{2}<\infty
$$

In our case, taking into account the explicit form of the determinant of the metric tensor leads to the constraints for each angular momentum $l$

$$
\begin{aligned}
& \int_{R}^{\infty} d r r\left(r^{2}\right)^{-l-2}<\infty \\
& \int_{0}^{R} d r \frac{1}{r^{3}}\left(r^{2}\right)^{-l+2}<\infty
\end{aligned}
$$

We observe that the modes with $l= \pm 1$ cannot fulfill both (34) and (35). Their norm will only show however a mild logarithmic divergence as a function of the size of large but finite systems. This will still make possible to find a signature of these modes, when looking for localized states in the numerical study of large graphene wormholes.

The above discussion applies to the particular case of taking $\Psi_{A}^{ \pm}=0$. It is clear however that similar solutions can be obtained by replacing the lower by the upper component of the Dirac spinor, while inverting the direction of the fictitious flux and the angular momentum number, as these combined operations represent an exact symmetry of the Dirac equation. We see therefore that the above localized states have their respective partners with $\Psi_{A}^{+} \neq 0$, corresponding again to angular momentum $l=0$ and \pm 1 .

A similar analysis can be carried out for wormhole geometries with $(6 n, 0)$ nanotubes in which $n$ is not a multiple of 3 . In these cases, the effective flux from the topological defects is reduced down to $\Phi=\pi$. Taking first $\Psi_{A}^{ \pm}=0$, the Dirac equation for the zero-energy modes becomes now

$$
\left(\partial_{r}-\frac{1}{r} i \partial_{\theta} \mp \frac{1}{2 r}+\frac{1}{2 r}\right) \Psi_{B}^{ \pm}=0 \quad \text { for } r \geqslant R
$$

and

$$
\left(\partial_{r}-\frac{1}{r} i \partial_{\theta} \pm \frac{1}{2 r}-\frac{1}{2 r}\right) \Psi_{B}^{ \pm}=0 \quad \text { for } r \leqslant R
$$

We find now more tight conditions to ensure the decay of the eigenmodes at $r \rightarrow \infty$ and $r \rightarrow 0$, as the resolution of the above equations gives the behaviors

$$
\begin{aligned}
& \Psi_{B}^{-} \sim r^{-l-1} e^{i l \theta}, \quad r \geqslant R \\
& \Psi_{B}^{-} \sim r^{-l+1} e^{i l \theta}, \quad r \leqslant R
\end{aligned}
$$

It turns out that, in this case, a localized state exists only for $l=0$. Its norm is not convergent in the infinite wormhole geometry but, as also happened in the preceding discussion, it just shows a soft logarithmic divergence that can make the mode to appear as a localized state in finite real systems. We complete this analysis by remarking that a similar mode arises, now with $\Psi_{A}^{+} \neq 0$, when the orientation of the fictitious gauge flux is inverted in the Dirac equation. 
The above search of the zero-energy modes shows that the number of localized states of the Dirac equation bears a direct relation to the gauge flux threading the two-dimensional system. This property is at the basis of the development of the Landau levels in a conventional transverse magnetic field, and it is confirmed in our system with fictitious gauge flux attached to the topological defects. The number of zero modes is actually a topological invariant, not sensitive to the particular details of the flux over the two-dimensional geometry. This is why their signatures can be found in the real carbon lattices where the effect of the fictitious gauge flux is not felt isotropically, as we will see in the numerical study of the graphene wormholes.

\section{Numerical approach to graphene wormhole spectra}

We want to check that the above features derived from the Dirac equation in the curved space are actually present in real graphene wormholes. For this purpose, we will adopt a numerical tight-binding approach to describe the electronic structure of the curved carbon material. Thus, we will assume that the electronic properties can be modeled by the hopping of electrons between nearest-neighbor atoms of the carbon lattice, following the same approach leading to Eq. (14). The tight-binding Hamiltonian reads now

$$
H=-t \sum_{\langle i, j\rangle} c_{i}^{\dagger} c_{j}
$$

where $c_{i}^{\dagger}\left(c_{i}\right)$ are electron creation (annihilation) operators and the sum runs over pairs of nearestneighbor carbon atoms in the graphene wormhole. The lattices we are going to consider are obtained by matching two nanotube-graphene junctions of the type shown in Fig. 1(c). This means that we will always have a short zig-zag nanotube bridge connecting the two graphene branches of the wormhole. We will take however this nanotube with a length much smaller than its radius, so that a sensible comparison can be achieved with the previous analytic approach to the wormhole in the continuum limit.

We have carried out the numerical diagonalization of the Hamiltonian (40) in lattices containing of the order of $\sim 100000$ atoms, for different values of the wormhole radius. In practice, we have matched the two nanotube-graphene junctions of the wormhole by aligning pairwise the position of heptagonal carbon rings at the two ends of the nanotube bridge. This choice has the advantage of preserving in the wormhole the symmetry of the nanotube-graphene junctions under rotations around the nanotube axis. In this way, it is possible to classify the different energy levels according to their eigenvalue $q$ under a rotation of $\pi / 3$. The spectrum can be decomposed then in several sectors corresponding to the different values $q=1, e^{ \pm i \pi / 3}, e^{ \pm 2 i \pi / 3}$ and -1 . This turns out to be very convenient for the purpose of drawing the connection with the eigenmodes having well-defined angular momentum in the continuum limit.

The inspection of spectra of different graphene wormholes shows that they fall into two broad classes, paying attention to the electronic structure in the different sectors labeled by the eigenvalue $q$. In this respect, a convenient way of characterizing the electronic system is to look at the features produced by the wormhole bridge, as this is the source of new electronic properties. For each wormhole, we have then computed preferentially the local density of states for a circular ring of atoms around one of the ends of the nanotube bridge. This has been complemented with the analysis of the spatial decay of the eigenmodes in the graphene sheets, in order to discern the propagating or localized character of the electronic states.

We show for instance in Fig. 5 the mentioned local density of states $\rho(\varepsilon)$ for a graphene wormhole with a short zig-zag $(54,0)$ nanotube bridge. The length of the bridge amounts to 


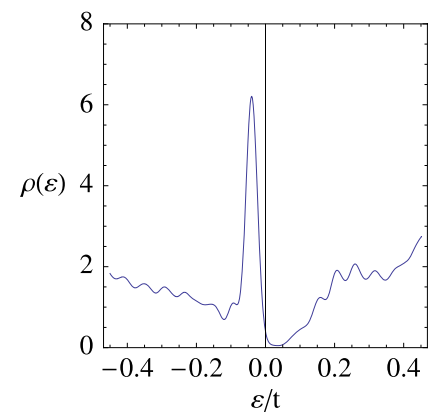

(a)

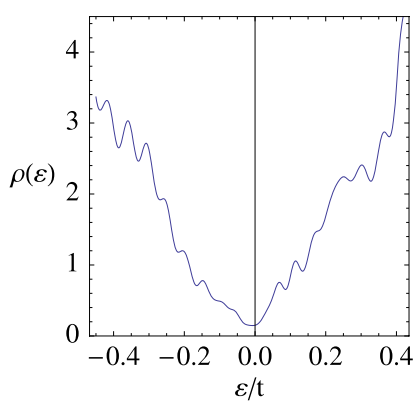

(c)

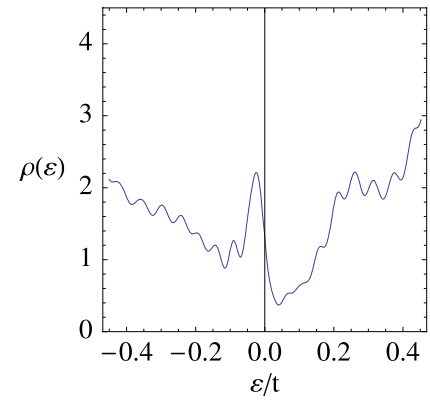

(b)

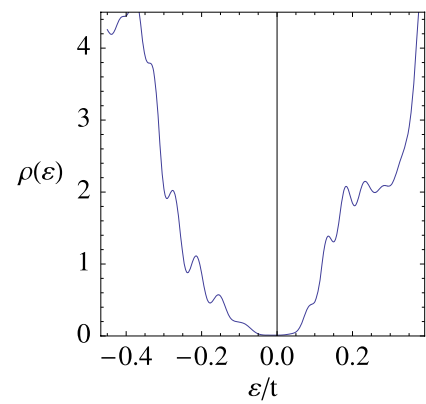

(d)

Fig. 5. Sequence of local densities of states for a circular ring of atoms around one of the ends of a wormhole bridge made of a short zig-zag $(54,0)$ nanotube, for the different sectors corresponding to eigenvalue $q$ under $\pi / 3$ rotation equal to 1 (a), $e^{ \pm i \pi / 3}$ (b), $e^{ \pm 2 i \pi / 3}$ (c), and -1 (d). Energy is measured in units of the transfer integral $t$.

two nanotube unit cells in this case, complying therefore with the requirement of being much smaller than the radius of the wormhole. We observe that the density of states is in general suppressed around $\varepsilon=0$, while there are prominent peaks at very low energy in the sectors corresponding to $q=1$ and $e^{ \pm i \pi / 3}$. The depletion in the density of states is consistent with the fact that the underlying effective theory in the continuum limit must be that of a pair of Dirac fermion fields, which have in general a spectrum with a vanishing density of states at the Dirac point. Furthermore, we have to bear in mind that, in the representations of the density of states, the contribution of each level has been smoothed with a Gaussian broadening in order to give a continuous appearance to the plots. Thus, a closer look reveals that a couple of almost degenerated levels is responsible for the peak in the sector $q=1$, while that for $q=e^{i \pi / 3}$ (or $q=$ $e^{-i \pi / 3}$ ) comes essentially from the contribution of two other levels with very low energy. Further analysis shows that the corresponding spatial distribution for these modes has a maximum at the wormhole bridge, with a pronounced decay when moving away along the radial direction in each graphene sheet.

We have checked that the peaks in the local density of states for $q=1$ and $e^{ \pm i \pi / 3}$ are always present when the graphene wormholes have a $(6 n, 0)$ nanotube bridge such that $n$ is a multiple of 3. Quite remarkably, these are the instances in which the fictitious gauge flux from the heptagonal carbon rings reaches its maximum value, leading to two triplets of zero-energy modes with angular momenta $l=0$ and \pm 1 in the effective continuum theory. This is then in agreement with the observation of the localized states in the numerical diagonalization of the graphene worm- 


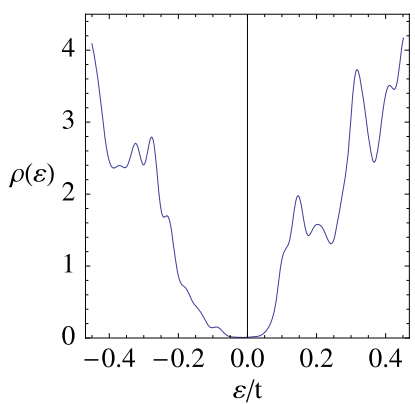

(a)

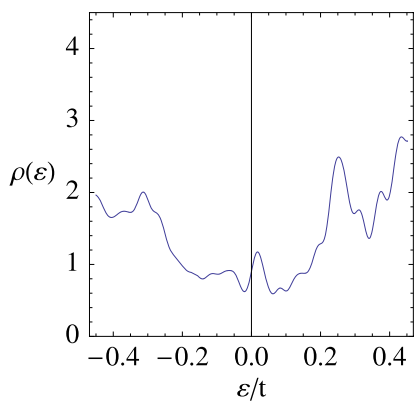

(c)

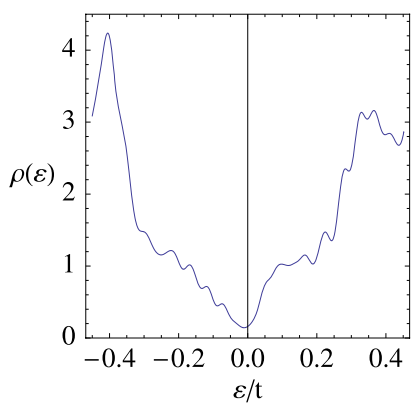

(b)

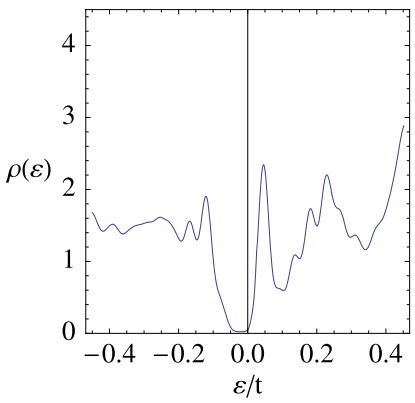

(d)

Fig. 6. Similar sequence as in Fig. 5 for a wormhole bridge made of a short zig-zag $(48,0)$ nanotube, for $q=1$ (a), $e^{ \pm i \pi / 3}(\mathrm{~b}), e^{ \pm 2 i \pi / 3}(\mathrm{c})$, and $-1(\mathrm{~d})$.

holes. We recall that, in the effective theory, the angular momentum $l$ gives the smooth spatial modulation corresponding to the envelope of the electron wavefunction. This has in general fast oscillations dictated by the large momenta $p_{x}= \pm 4 \pi / 3 \sqrt{3} a$ at the Dirac points [15]. In the case of zig-zag $(6 n, 0)$ nanotubes with $n$ equal to a multiple of 3 , however, the oscillations in the lowest-energy branches of the spectrum turn out to correspond to the eigenvalue $q=1$. This explains why the localized states with $l=0$ and \pm 1 appear in the lattice for such a value of $q$ and those obtained by adding or subtracting one unit of angular momentum.

The rest of graphene wormholes, with a zig-zag $(6 n, 0)$ nanotube bridge where $n$ is not a multiple of 3, display spectra with a different but distinctive behavior. This is illustrated in Fig. 6 in the case of a wormhole made of a short $(48,0)$ nanotube. The local density of states $\rho(\varepsilon)$ is again depleted in general around $\varepsilon=0$, but it does not vanish now in the sectors with $q=e^{ \pm 2 i \pi / 3}$, showing even a small peak at very low energy. This can be explained by the fact that, in the $(6 n, 0)$ nanotubes with $n$ not equal to a multiple of 3 , the lowest-energy branches have in general large angular momentum numbers that do not correspond to the eigenvalue $q=1$. In the zigzag nanotubes, the electrons pick up a large transverse momentum $p_{\perp}=4 \pi / 3 \sqrt{3} a$ at the Dirac point, which translates when turning around the nanotube waist of length $6 \sqrt{3} n a$ into a variation of the phase $\Delta \phi=8 \pi n$ for the electron wavefunction. If $n$ is not a multiple of 3 , the symmetry of these lowest-energy states under a rotation of $\pi / 3$ makes them to fall always in the sector with $q=e^{ \pm 2 i \pi / 3}$. It seems therefore that, though the nanotube bridge has a short length in our lattices (extending just four nanotube unit cells in the example of Fig. 6), it plays a crucial role dictating the symmetry of the low-energy states in the wormhole. 
It can be checked that the small peak in the sector with $q=e^{2 i \pi / 3}$ in Fig. 6 (and its counterpart for $q=e^{-2 i \pi / 3}$ ) arises from the presence of a state with very low energy localized at the wormhole. This agrees with the prediction of the effective continuum theory developed above, bearing in mind that the states with $l=0$ have their spatial oscillations dictated by the large momentum at the Dirac point, which leads in this case to $q=e^{ \pm 2 i \pi / 3}$. The smallness of the peak is partly due to the fact that it comes from the contribution of a single level for the given value of $q$, but it can be also taken as a reflection that the localized mode cannot give rise to a truly normalizable state in the continuum theory. On the other hand, we remark that other low-energy features like the innermost peaks at both sides of the Fermi level in Fig. 6(d) have to do instead with the resonant transmission through the nanotube bridge. This is consistent with the fact that similar peaks have been already observed in that sector of the local density of states in carbon nanotube-graphene junctions [16]. The presence of the nanotube bridge is in general responsible for those sharp enhancements in the local density of states, which mark the thresholds at which new channels open for transmission.

Going back to Fig. 5, we observe that the much larger peak found in the sector with $q=1$, in comparison to those for $q=e^{ \pm i \pi / 3}$ in that figure, is actually the signature of the strong localization of a couple of states in the graphene wormhole, in agreement with the prediction of two well-defined bound states in the continuum theory. This further supports the correspondence between the low-energy features observed in the numerical diagonalization of the tight-binding Hamiltonian and the results from the effective Dirac theory, confirming the validity of the latter for the continuum limit description of the curved carbon lattices.

\section{Conclusions}

In this paper we have developed an effective field theory accounting for the continuum limit of graphene wormholes, when their radius is much larger than the length of the nanotube bridge. Under this condition, we have dealt with a minimal continuum model of the wormhole geometry obtained by matching two two-dimensional sheets at the boundary of a common circular hole. This provides a sensible description of the properties of the lattice geometry when looking over large length scales, as the graphene wormholes have their intrinsic curvature concentrated on 12 heptagonal carbon rings at the junctions of the graphene branches with the nanotube bridge. A nice check of the consistency of the continuum model is made by realizing that the integral of the curvature over the two-dimensional space is an integer number (in units of $4 \pi$ ) matching the value expected from the sum of the contributions of the heptagonal carbon rings in the lattice geometry.

We have seen that the continuum limit has to be complemented by including the effect that the heptagonal carbon rings induce on the Dirac fields encoding the low-energy electronic excitations of the carbon material. This action can be mimicked by attaching a line of fictitious gauge flux at each topological defect, as already illustrated in the case of the fullerene lattices [15]. The graphene wormholes represent actually an instance which is to some extent dual to the case of the fullerenes, as the 12 pentagonal carbon rings in those closed lattices play a role opposite to that of the heptagonal defects in the wormhole. It is remarkable that the zero-energy modes of the Dirac equation in the continuum model of the wormhole can be arranged into two triplets, when the fictitious gauge flux traversing the two-dimensional space becomes maximal (and actually equal to the radial flux supported by the fullerene molecules). We can think of those low-lying levels as the counterpart of the two well-known triplets of zero modes that arise in the electronic spectra of the fullerenes in the continuum limit. Beyond that similarity, it is clear that the results 
from our effective Dirac theory are a particular realization of the correspondence between the number of zero modes of the Dirac equation and the value of the gauge flux traversing the twodimensional space.

With our numerical investigation of the electronic spectra of graphene wormholes, we have seen that these may fall into two different classes, depending on whether the $(6 n, 0)$ nanotube bridge corresponds to $n$ being a multiple of 3 or not. The numerical computations performed in the tight-binding approach have shown that two triplets of localized states appear in the wormholes where $n$ is equal to a multiple of 3 , while in the rest of the cases there are two localized states at very low energies. This turns out to be in agreement with the analysis establishing that the effective gauge flux from a combination of topological defects depends in general on their relative location. Thus, only when the separation between two heptagonal carbon rings is given by a vector $(N, M)$ (in units of the translation vectors of the hexagonal lattice) such that $N-M$ is a multiple of 3 , the effective flux from the pair of heptagons corresponds to the sum of the individual fluxes [19]. This count can be used to show that the predictions of the number of localized modes from the effective Dirac theory are consistent with the two different classes of electronic spectra displayed in the numerical diagonalization of the tight-binding Hamiltonians.

In this paper we have focused on the analysis of graphene wormholes where the heptagonal carbon rings are regularly distributed around each end of the nanotube bridge. Such a constraint leads to wormhole geometries in which the graphene branches are connected by zig-zag $(6 n, 0)$ or armchair $(6 n, 6 n)$ nanotubes. This can be already realized from the study of the carbon nanotube-graphene junctions carried out in Ref. [16]. We have checked that the graphene wormholes with armchair bridge not considered here fall actually in the same class mentioned above with the two triplets of localized states at very low energy. This is again consistent with the rule giving the effective gauge flux of a combination of topological defects, which leads to the maximal value for a wormhole with $(6 n, 6 n)$ bridge as the separation between heptagonal rings at each end is given then by a vector $(n, n)$. It is also possible to think of more general graphene wormholes in which the topological defects do not have a regular distribution. In these cases, we expect anyhow that the number of localized states will be dictated again by the effective gauge flux going through the two-dimensional space.

For practical purposes, the most significant achievement of our investigation has been to show that the low-energy electronic properties of the graphene wormholes are consistently described by an effective theory of two Dirac fermion fields in the curved geometry of the wormhole. This may open the way to use real samples of the curved carbon material as a playground to experiment with the interaction between the background curvature and the Dirac fields. In this regard, we recall that carbon nanotube-graphene junctions have been already produced in the laboratory [21], and it is also conceivable that graphene wormholes may be fabricated starting from the easily available graphene bilayers. The investigation of the condensed matter system can make then possible to study relevant gravitational effects related to the Dirac character of the electron quasiparticles, which otherwise would be only accessible at the much higher energies typical of the astrophysical phenomena.

\section{Acknowledgements}

We thank F. Barbero, F. Guinea and E.J.S. Villaseñor for very fruitful discussions. The financial support from MICINN (Spain) through grant FIS2008-00124/FIS is gratefully acknowledged. 


\section{References}

[1] K.S. Novoselov, A.K. Geim, S.V. Morozov, D. Jiang, Y. Zhang, S.V. Dubonos, I.V. Grigorieva, A.A. Firsov, Science 306 (2004) 666.

[2] K.S. Novoselov, A.K. Geim, S.V. Morozov, D. Jiang, M.I. Katsnelson, I.V. Grigorieva, S.V. Dubonos, A.A. Firsov, Nature 438 (2005) 197.

[3] Y. Zhang, Y.-W. Tan, H.L. Stormer, P. Kim, Nature 438 (2005) 201.

[4] A.H. Castro Neto, F. Guinea, N.M.R. Peres, K.S. Novoselov, A.K. Geim, Rev. Mod. Phys. 81 (2009) 109.

[5] H. Suzuura, T. Ando, Phys. Rev. Lett. 89 (2002) 266603.

[6] M.I. Katsnelson, K.S. Novoselov, A.K. Geim, Nature Phys. 2 (2006) 620.

[7] M.M. Fogler, D.S. Novikov, B.I. Shklovskii, Phys. Rev. B 76 (2007) 233402.

[8] A.V. Shytov, M.I. Katsnelson, L.S. Levitov, Phys. Rev. Lett. 99 (2007) 236801

[9] V.M. Pereira, V.N. Kotov, A.H. Castro Neto, Phys. Rev. B 78 (2008) 085101.

[10] I.S. Terekhov, A.I. Milstein, V.N. Kotov, O.P. Sushkov, Phys. Rev. Lett. 100 (2008) 076803.

[11] J. González, F. Guinea, M.A.H. Vozmediano, Nucl. Phys. B 424 (1994) 595.

[12] J. González, F. Guinea, M.A.H. Vozmediano, Phys. Rev. B 59 (1999) R2474.

[13] S. Das Sarma, E.H. Hwang, W.-K. Tse, Phys. Rev. B 75 (2007) 121406(R).

[14] M.I. Katsnelson, A.K. Geim, Philos. Trans. R. Soc. A 366 (2008) 195.

[15] J. González, F. Guinea, M.A.H. Vozmediano, Nucl. Phys. B 406 (1993) 771.

[16] J. González, F. Guinea, J. Herrero, Phys. Rev. B 79 (2009) 165434.

[17] J. González, F. Guinea, M.A.H. Vozmediano, Phys. Rev. Lett. 69 (1992) 172.

[18] R. Saito, G. Dresselhaus, M.S. Dresselhaus, Physical Properties of Carbon Nanotubes, Imperial College Press, London, 1998.

[19] P.E. Lammert, V.H. Crespi, Phys. Rev. B 69 (2004) 035406.

[20] N.D. Birrell, P.C.W. Davies, Quantum Fields in Curved Space, Cambridge Univ. Press, Cambridge, 1982.

[21] See http://jp.fujitsu.com/group/labs/downloads/en/business/activities/activities-3/fujitsu-labs-nanotech-001-en.pdf. 